\title{
A Systematic Review on Fake News Themes Reported in Literature
}

\author{
Marlie Celliers $^{(\varpi)}([)$ and Marie Hattingh $[1]$ \\ Department of Informatics, University of Pretoria, Pretoria, South Africa \\ marliecelliers22@gmail.com, marie.hattingh@up.ac.za
}

\begin{abstract}
In this systematic literature review, a study of the factors involved in the spreading of fake news, have been provided. In this review, the root causes of the spreading of fake news are identified to reduce the encouraging of such false information. To combat the spreading of fake news on social media, the reasons behind the spreading of fake news must first be identified. Therefore, this literature review takes an early initiative to identify the possible reasons behind the spreading of fake news. The purpose of this literature review is to identify why individuals tend to share false information and to possibly help in detecting fake news before it spreads.
\end{abstract}

Keywords: Fake news $\cdot$ Misinformation $\cdot$ Social media $\cdot$ Systematic review

\section{Introduction}

The increase in use of social media exposes users to misleading information, satire and fake advertisements [3]. Fake news or misinformation is defined as fabricated information presented as the truth [6]. It is the publication of known false information and sharing it amongst individuals [7]. It is the intentional publishing of misleading information and can be verified as false through fact-checking [2]. Social media platforms allow individuals to fast share information with only a click of a single share button [4]. In previous studies the effect of the spreading and exposure to misleading information have been investigated [6]. Some studies determined that everyone has problems with identifying fake news, not just users of a certain age, gender or education [11]. The literacy and education of fake news is essential in the combating of the spreading of false information [8].

This review identify and discuss the factors involved in the sharing and spreading of fake news. The outcome of this review should be to equip users with the abilities to detect and recognise misinformation and also to cultivate a desire to stop the spreading of false information [5]. 


\section{Literature Background}

\subsection{The Impact of Fake News}

The internet is mainly driven by advertising [13]. Websites with sensational headlines are very popular, which leads to advertising companies capitalising on the high traffic to the site [13]. It was subsequently discovered that the creators of fake news websites and information could make money through automated advertising that rewards high traffic to their websites [13]. The question remains how misinformation would then influence the public. The spreading of misinformation can cause confusion and unnecessary stress among the public [10]. Fake news that is purposely created to mislead and to cause harm to the public is referred to as digital disinformation [17]. Disinformation has the potential to cause issues, within minutes, for millions of people [10]. Disinformation has been known to disrupt election processes, create unease, disputes and hostility among the public [17].

\subsection{Fake News and Social Media}

These days, the internet have become a vital part of our daily lives [2]. Traditional methods of acquiring information have nearly vanished to pave the way for social media platforms [2]. It was reported in 2017 that Facebook was the largest social media platform, hosting more 1.9 million users world-wide [18]. The role of Facebook in the spreading of fake news possibly has the biggest impact from all the social media platforms [14]. It was reported that $44 \%$ of worldwide users get their news from Facebook [14]. 23\% of Facebook users have indicated that they have shared false information, either knowingly or not [19]. The spreading of fake news is fuelled by social media platforms and it is happening at an alarming pace [14].

\section{Research Method}

In this systematic literature review, a qualitative methodology was followed. A thematic approach was implemented to determine the factors and sub-factors that contribute to the sharing and spreading of fake news. The study employed the following search terms: ("Fake News" (NEAR/2) "Social Media") AND (defin* OR Factors OR Tools) ("Misinformation" (NEAR/2) "Social Media") AND (defin* OR Factors OR Tools).

In this literature review, only published journal articles between 2016 and 2019 were considered. This review is not be specific to certain sectors i.e. the health sector or the tourism sector but rather consider on all elements that contribute to individuals sharing false information.

Studies that are not in English have been excluded in this review. Only studies that are related to the research question have been taken into account. This article does not discuss the detection of fake news but rather the reasons behind the spreading of fake news. 
The analysis consisted of four phases: identification phase; screening phase; eligibility phase and inclusion phase. When conducting this literature review, the selection of articles were based on three main criteria: firstly, to search for and select articles containing the search terms identified above; secondly, selection based on the title and abstract of the article and finally selection based on the content of the article.

In the identification phase of this literature review, Science Direct and Emerald Insight were selected to perform the literature review. Science Direct offered a total of 177 journal articles matching the search terms. Emerald Insight generated 121 journal articles that matched the search terms. Continuing with the identification phase, the various articles were then combined and the duplicates were removed. In the screening phase of the source selection, all the article titles were carefully screened and a few articles were excluded as unconvincing. The eligibility of the abstract in the remaining articles were consulted and some articles were excluded based on the possible content of the article. The rest of the articles were further thoroughly examined to determine if they were valuable and valid to this research paper. Upon further evaluation, these final remaining articles were further studied to make a final source selection.

\section{Analysis of Findings}

In this paper, possible reasons for and factors contributing to the sharing and spreading of false information are discussed. The reasons are categorized under various factors highlighted in the journal articles used to answer the research question. These factors include: social factors, cognitive factors, political factors, financial factors and malicious factors.

While conducting the literature review, 22 articles highlighted the social factors; 13 articles discussed the role that cognitive factors have in contributing to the sharing and spreading of fake news; 13 articles highlighted the role of political factors; nine articles discussed how financial gain could convince a social media users to spread false information and 13 articles debated malicious factors and the effect that malicious factors have on the sharing and spreading of false information.

Figure 1 gives a breakdown of all 38 articles containing references to all the subcategories listed above. It was clearly evident that the two single sub-categories of social comparison and hate propaganda were the most debated. With the sub-factor, knowledge and education, closely behind. A high percentage of the articles, 34.2\% (13 of 38), refer to the effects of social comparison on the spreading of false information; followed by $26.3 \%$ (10 of 38) of the articles referencing hate propaganda. Knowledge and education was measured at $23.6 \%$ (9 of 38 ).

Furthermore, it was concluded that the majority of the 38 articles highlighted a combination of the social factors i.e. conformity and peer influence, social comparison and satire and humorous fakes, which measures at $60.5 \%$ (23 of 38). Where the combination of the cognitive factors e.g. knowledge and education and ignorance measured at 39.4\% (15 of 38). Political factors and sub-factors e.g. political clickbaits and political bots/cyborgs, were discussed in 34.2\% (13 of 38) of the articles. In 
addition, financial factors e.g. advertising and financial clickbaits were referenced in $23.6 \%$ ( 9 of 38) of the journal articles. And lastly, malicious factors e.g. malicious bots and cyborgs, hate propaganda and malicious clickbaits measured at 34.2\% (13 of 38).

\section{Sub-Categories of the Spreading of Fake News}

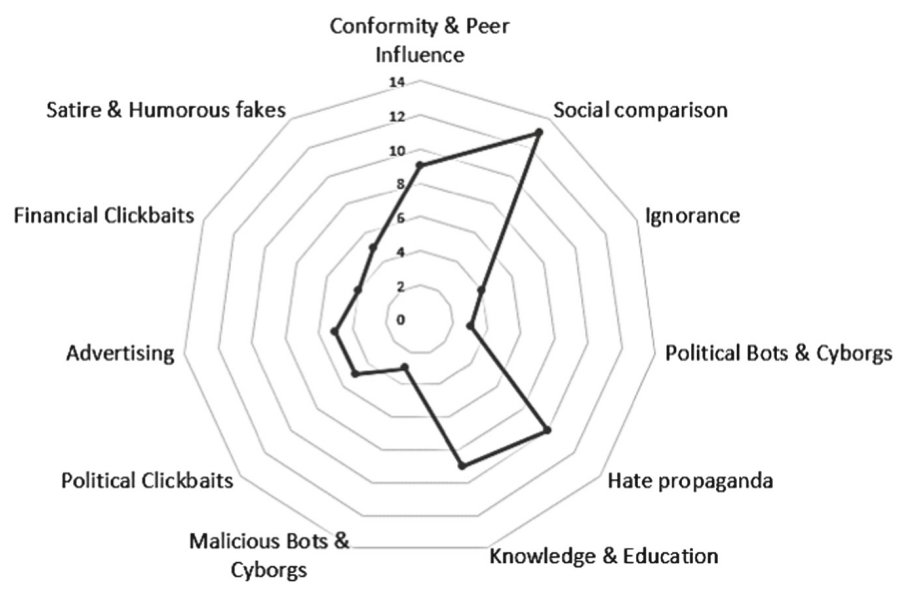

Fig. 1. Articles discussing the sub-categories of the spreading of fake news

\section{Discussion}

\subsection{Social Factors}

Fake news stories are being promoted on social media platforms to deceive the public for ideological gain [20]. In various articles it was stated that social media users are more likely to seek information from people who are more like-minded or congruent with their own opinions and attitudes [21, 22].

Conformity and Peer Influence. It is the need of an individual to match his or her behaviour to a specific social group [9]. The desire that social media users have to enhance themselves on social media platforms could blur the lines between real information and false information [12]. Consequently, social media users will share information to gain social approval and to build their image [12]. Recent studies have shown that certain false information can be strengthened if it belongs to the individuals in the same social environment [23]. The real power lies with those certain individuals who are more vocal or influential [24]. The need for social media users to endorse information or a message can be driven by the perception the social media user has about the messenger [25]. These messengers or "influencers" can be anyone ranging from celebrities to companies [24].

Studies show that messages on social media platforms, like Twitter, gain amplification because the message or information is associated with certain users or influencers [25]. Information exchanging depends on the ratings or the influential users 
associated with the information [23]. Social media users' influence among peers enhance the impact and spreading of all types of information [25]. These social media influencers have the ability to rapidly spread information to numerous social media users [26]. The level of influence these influencers have, can amplify the impact of the information [27].

The lack of related information in online communities could lead to individuals sharing the information based on the opinions and behaviours of others [23]. Some studies show that social media users will seek out or share information that reaffirms their beliefs or attitudes [18].

Social Comparison. The whole driving force of the social media sphere is to post and share information [1]. Social comparison can be defined as certain members within the same social environment who share the same beliefs and opinions [12]. When they are unable to evaluate certain information on their own, they adapt to compare themselves to other members, within the same environment, with the same beliefs and opinions [12]. The nature of social media allows social media users to spread information in realtime [28]. Social media users generate interactions on social media platforms to gain "followers" and to get "likes" which lead to an increasing amount of fake news websites and accounts [10]. One of the biggest problems faced in the fake news dilemma, is that social media users' newsfeed on social media platforms, like Facebook, will generally be populated with the user's likes and beliefs, providing a breeding ground for users with like-minded beliefs to spread false information among each other [16]. Social media users like to pursue information from other members in their social media environment whose beliefs and opinions are most compatible with their own [21].

Social media algorithms designed to make suggestions or filter information based on the social media users' preferences [29]. The "like" button on social media platforms, e.g. Facebook, becomes a measuring tool for the quality of information, which could make social media users more willing to share the information if the information has received multiple likes [30]. Social media users' belief in certain information depends on the number of postings or "re-Tweets" by other social media users who are involved in their social media sphere [23]. One article mentioned that the false news spreading process can be related to the patterns of the distribution of news among social media users [31]. The more a certain piece of information is shared and passed along the more power it gains [6].

This "endorsing" behaviour results in the spreading of misleading information [25]. It is also known as the "herding" behaviour and is common among social media where individuals review and comment on certain items [23]. It is also referred to as the "bandwagon effect" where individuals blindly concentrate on certain information based on perceived trends [32]. The only thing that matters is that the information falls in line with what the social media user wants to hear and believe [16]. Many studies also refer to it as the "filter bubble effect" where social media users use social media platforms to suggest or convince other social media users of their cause [33]. Communities form as a result of these filter bubbles where social media users cut themselves off from any other individual that might not share the same beliefs or opinions [33]. It was found that social media users tend to read news or information that are ideologically similar to their own ideologies [29]. 
Satire and Humorous Fakes. Some of the content on social media are designed to amuse users and are made to deceive people into thinking that it is real news [2]. Satire is referred to as criticising or mocking ideas or opinions of people in an entertaining or comical way [7, 29]. These satire articles consist of jokes or forms of sarcasm that can be written by everyday social media users [10]. Most satire articles are designed to mislead and instruct certain individuals [13]. Some social media users will be convinced that it is true information and will thus share the information [2].

\subsection{Cognitive Factors}

The study of cognition is the ability of an individual to make sense of certain topics or information by executing a process of reasoning and understanding [34]. It is the ability of an individual to understand thought and execute valid reasoning and understanding of concept [34]. With an increasing amount of information being shared across social media platforms it can be challenging for social media users to determine which of the information is closest to the original source [22]. The issue of individuals not having the ability to distinguish between real and fake news have been raised in many articles [10]. Users of social media tend to not investigate the information they are reading or sharing [2]. This can therefore lead to the rapid sharing and spreading of any unchecked information across social media platforms [2].

Knowledge and Education. An important aspect of surfing social media is the ability of the social media users to distinguish between what is real and what is fake [10]. The trustworthiness of a certain article is based on how successful the exchange of the articles are [12]. The more successful the exchange, the more likely social media users will share the information [12]. Social media users make supposedly reasonable justifications to determine the authenticity of the information provided [35].

People creating fake news websites and writing false information exploit the nonintellectual characteristics of some people [13]. For social media users to determine if the information they received is true or false, expert judgement of content is needed [1].

In a recent study, it was found that many social media users judge the credibility of certain information based on detail and presentation, rather than the source [11]. Some individuals determine the trustworthiness of information provided to them through social media on how much detail and content it contains [11].

It is believed that people are unable to construe information when the information given to them, are conflicting with their existing knowledge base [34]. Most social media users lack the related information to make a thorough evaluation of the particular news source [23]. For many years, companies and people have been creating fake news articles to capitalize on the non-intellectual characteristics of certain individuals [13].

Ignorance. A driving force of the spreading of false information is that social media users undiscerningly forward false information [5] A reason for the spreading of false information in many cases are inattentive individuals who do not realise that some websites mimic real websites [2]. These false websites are designed to look like the real website but in essence only contain false information. Social media users tend to share information containing a provocative headline, without investigating the facts and sources [16]. The absence of fact-checking by social media users on social media 
platforms, increases the spreading of false information [2]. Social media users tend to share information without verifying the source or the reliability of the content [2]. Information found on social media platforms, like Twitter, are sometimes not even read before they are being spread among users, without any investigation into the source of the information [16]. As mentioned earlier in Sect. 5.1, the bandwagon effect causes individuals to share information without making valued judgement [32].

\subsection{Political Factors}

The spreading of false political information have increased due to the emergence of streamline media environments [22]. There has been a considerable amount of research done on the influence of fake news on the political environment [14, 18]. By creating false political statements, voters can be convinced or persuaded to change their opinions [3]. Critics reported that in the national election in the UK (regarding the nation's withdrawal out of the EU) and the 2016 presidential election in the US, a number of false information was shared on social media platforms that have influenced the outcome of the results $[8,22]$. Social media platforms, like Facebook, came under fire in the 2016 US presidential election, when fake news stories from unchecked sources were spread among many users [10]. The spreading of such fake news have the sole purpose of changing the public's opinion [8, 29].

Various techniques can be used to change the public's opinion. These techniques include repeatedly retweeting or sharing messages often with the use of bots or cyborgs [15]. It also includes misleading hyperlinks that lures the social media user to more false information [15].

Political Clickbaits. Clickbaits are defined as sources that provide information but use misleading and sensational headiness to attract individuals [16]. In the 2016 US presidential elections it was apparent that clickbaits were used to shape peoples' opinions [4]. In a recent study it was found that 43\% (13 of 30) false news stories were shared on social media platforms, like Twitter, with links to non-credible news websites [22].

Webpages are purposely created to resemble real webpages for political gain [3]. News sources with URLs similar to the real website URL have been known to spread political fake news pieces, which could influence the opinion of the public [10].

Political Bots/Cyborgs. A social media users' content online is managed by algorithms to reflect his or her prior choices [22]. Algorithms designed to fabricate reports are one of the main causes of the spreading of false information [33]. In recent years, the rapid growth of fake news have led to the belief that cyborgs and bots are used to increase the spreading of misinformation on social media [22]. In the 2016 US election social bots were used to lead social media users to fake news websites to influence their opinions on the candidates [3]. Hundreds of bots were created in the 2016 US presidential elections to lure people to websites with false information [3] These social bots can spread information through social media platforms and participate in online social communities [3]. 


\subsection{Financial Factors}

One of the biggest problems with fake news is that it allows the writers to receive monetary incentives [13]. Misleading information and stories are promoted on social media platforms to deceive social media users for financial gain [20, 29].

One of the main goals of fake news accounts are to generate traffic to their specific website [10]. Articles with attractive headlines lure social media users to share false information thousands of times [2]. Many companies use social media as a platform to advertise their products or to promote their products [26].

Advertising. People earn money through clicks and views [36]. The more times the link is clicked the more advertising money is generated [16]. Every click corresponds to advertising revenue for the content creator [16]. The more traffic companies or social media users get to their fake news page, the more profit through advertising can be earned [10]. The only way to prevent financial gain for the content creator is inaction [16]. Most advertising companies are more interested in how many social media users will be exposed to their product rather than the possible false information displayed on the page where their advertisement is displayed [13]. Websites today are not restricted on the content displayed to the public, as long as they attract users [13]. This explains how false information is monetized, providing monetary value for writers to display sensational false information [13].

Financial Clickbaits. Clickbaits are used to lure individuals to other websites or articles for financial gain [2]. One of the main reasons for falsifying information is to earn money through clicks and views [36]. Writers focus on sensational headlines rather than truthful information [13]. Appeal rather than truthfulness drives information [5]. These attractive headlines deceive individuals into sharing certain false information [2].

Clickbaits are purposely implemented to misguide or redirect social media users to increase the views and web traffic of certain websites for online advertising earnings [4]. Social media users end up spending only a short time on these websites [4]. Clickbaits have been indicated as one of the main reasons behind the spreading of false information [2].

\subsection{Malicious Factors}

Studies debating the trustworthiness of information and veracity analytics of online content have increased recently due to the rise in fake news stories [37]. Social media has become a useful way for individuals to share information and opinions about various topics [3]. Unfortunately, many users share information with malicious intent [3]. Malicious users, also referred to as "trolls", often engage in online communication to manipulate other social media users and to spread rumours [37].

Malicious websites are specifically created for the spreading of fake news [2]. Malicious entities use false information to disrupt daily activities like the health-sector environment, the stock markets or even the opinions people have on certain products [3]. Some online fake news stories are purposely designed to target victims [3]. Websites, like Reddit, have been known as platforms where users can get exposed to 
bullying [38]. Some individuals have been known to use the social media platform to cause confusion and fear among others [39].

Malicious Bots/Cyborgs. Malicious users, with the help of bots, target absent-minded individuals who do not check the article facts or source before sharing it on social media [2]. These AI powered bots are designed to mimic human behaviour and characteristics, and are used to corrupt online conversations with unwanted and misleading advertisements [38]. In recent studies it was found that social bots are being created to distribute malware and slander to damage an individual's beliefs and trust [3].

Hate Propaganda. Many argue that the sharing of false information fuel vindictive behaviour among social media users [12]. Some fake news websites or pages are specifically designed to harm a certain user's reputation $[5,10]$. Social media influencers influence users' emotional and health outcomes [12]. Fake news creators specifically target users with false information [3]. This false information is specifically designed to deceive and manipulate social media users [21]. Fake news stories like this, intend to mislead the public and generate false beliefs [21]. In some cases, hackers have been known to send out fake requests to social media users to gain access to their personal information [39]. The spreading of hoax has also become a problem on social media. The goal of hoaxes is to manipulate the opinion of the public and to maximize public attention [35]. Social spammers have also become more popular over the last few years with the goal to launch different kinds of attacks on social media users, for example spreading viruses or phishing [2]. Fake reviews have also been known to disrupt the online community through writing reviews that typically aim to direct people away from a certain product or person [2]. Another method used by various malicious users, is to purchase fake followers to spread harmful malware more swiftly [26].

Malicious Clickbaits. It was reported on in a previous article that employees in a certain company clicked on a link, disguised as important information, where they provide sensitive and important information to perpetrators [4]. Malicious users intending to spread malware and phishing hide behind a fake account to further increase their activities [26]. Clickbaits in some cases are designed to disrupt interactions or to lure individuals into arguing in disturbed online interactions or communications [37]. These clickbaits have also been known to include malicious code as part of the webpage [4]. This will cause the social media users to download malware onto their device once they select the link [4].

\section{Conclusion}

Various articles were used to identify and study the factors and reasons involved in the sharing and spreading of misinformation on social media. Upon retrieving multiple reasons for the spreading of false information, they were categorized into main factors and sub-factors. These factors included social factors, cognitive factors, political factors, financial factors and malicious factors. Considering the rapidly expanding social media environment, it was found that especially social factors have a very significant influence in the sharing of fake news on social media platforms. Its sub-factors of 
conformity and peer-influence; social comparison and satire and humorous fakes have great influence when deciding to share false information. Secondly, it was concluded that malicious factors like hate propaganda also fuel the sharing of false information with the possibility to financially gain or to do harm.

In addition, it was concluded from this review that knowledge and education plays a very important role in the sharing of false information, where social media users sometimes lack the logic, reasoning and understanding of certain information. It was also evident that social media users may sometimes be ignorant and indifferent when sharing and spreading information. Fact-checking resources are available but the existence thereof is fairly unknown and therefore often unused. Hopefully better knowledge and education will encourage a desire among social media users to be more aware of possible unchecked information and the sources of information and to stop the forwarding of false information. A better understanding of the motives behind the sharing of false information can potentially prepare social media users to be more vigilant when sharing information on social media. The goal of this literature review was only to identify the factors that drive the spreading of fake news on social media platforms and did not fully address the dilemma of combatting the sharing and spreading of false information.

While this literature review sheds light on the motivations behind the spreading of false information, it does not highlight the ways in which one can detect false information. This proposes further suggestions for follow-up research or literature studies using these factors in an attempt to detect and limit or possibly eradicate the spreading of false information across social media platforms. Despite the limitations of this literature review, it helps to educate and provide insightful knowledge to social media users who share information across social media platforms.

\section{References}

1. Obelitz Søe, S.: Algorithmic detection of misinformation and disinformation: Gricean perspectives. J. Doc. 72(2), 309 (2018)

2. Bondielli, A., Marcelloni, F.: A survey on fake news and rumour detection techniques. Inf. Sci. 497, 38-55 (2019)

3. Zhang, X., Ghorbani, A.A.: An overview of online fake news: characterization, detection, and discussion. Inf. Process. Manag. 57(2), 102025 (2019)

4. Aldwairi, M., Alwahedi, A.: Detecting fake news in social media networks. Proc. Comput. Sci. 141, 215-222 (2018)

5. Chen, X., Sin, S.-C.J., Theng, Y.-L., Lee, C.S.: Why students share misinformation on social media: motivation, gender, and study-level differences. J. Acad. Librariansh. 41(5), 583-592 (2015)

6. Shin, J., Jian, L., Driscoll, K., Bar, F.: The diffusion of misinformation on social media: Temporal pattern, message, and source. Comput. Hum. Behav. 83, 278-287 (2018)

7. Gravanis, G., Vakali, A., Diamantaras, K., Karadais, P.: Behind the cues: a benchmarking study for fake news detection. Expert Syst. Appl. 128, 201-213 (2019)

8. Kanoh, H.: Why do people believe in fake news over the Internet? An understanding from the perspective of existence of the habit of eating and drinking. Proc. Comput. Sci. 126, 1704-1709 (2018) 
9. Colliander, J.: 'This is fake news': Investigating the role of conformity to other users' views when commenting on and spreading disinformation in social media. Comput. Hum. Behav. 97, 202-215 (2019)

10. Figueira, Á., Oliveira, L.: The current state of fake news: challenges and opportunities. Proc. Comput. Sci. 121, 817-825 (2017)

11. Atodiresei, C.-S., Tănăselea, A., Iftene, A.: Identifying fake news and fake users on Twitter. Proc. Comput. Sci. 126, 451-461 (2018)

12. Talwar, S., Dhir, A., Kaur, P., Zafar, N., Alrasheedy, M.: Why do people share fake news? Associations between the dark side of social media use and fake news sharing behavior. J. Retail. Consum. Serv. 51, 72-82 (2019)

13. Burkhardt, J.M.: History of fake news. Libr. Technol. Rep. 53(8), 5-9 (2017)

14. Anderson, K.E.: Getting acquainted with social networks and apps: combating fake news on social media. Libr. Hi Tech News 35(3), 1-6 (2018)

15. Al-Rawi, A., Groshek, J., Zhang, L.: What the fake? Assessing the extent of networked political spamming and bots in the propagation of \#fakenews on Twitter. Online Inf. Rev. 43(1), 53-71 (2019)

16. Rochlin, N.: Fake news: belief in post-truth. Libr. Hi Tech 35(3), 386-392 (2017)

17. IEC South Africa: Real411. Keeping it real in digital media. Disinformation Destroys Democracy (2019)

18. Lor, P.J.: Democracy, information, and libraries in a time of post-truth discourse. Libr. Manag. 39(5), 307-321 (2018)

19. Yu, F., Liu, Q., Wu, S., Wang, L., Tan, T.: Attention-based convolutional approach for misinformation identification from massive and noisy microblog posts. Comput. Secur. 83, 106-121 (2019)

20. Jang, S.M., Kim, J.K.: Third person effects of fake news: fake news regulation and media literacy interventions. Comput. Hum. Behav. 80, 295-302 (2018)

21. Buschman, J.: Good news, bad news, and fake news: going beyond political literacy to democracy and libraries. J. Docum. 75(1), 213-228 (2019)

22. Jang, S.M., et al.: A computational approach for examining the roots and spreading patterns of fake news: evolution tree analysis. Comput. Hum. Behav. 84, 103-113 (2018)

23. Wang, Q., Yang, X., Xi, W.: Effects of group arguments on rumor belief and transmission in online communities: an information cascade and group polarization perspective. Inf. Manag. 55(4), 441-449 (2018)

24. Lewandowsky, S., Ecker, U.K.H., Cook, J.: Beyond misinformation: understanding and coping with the 'Post-Truth' era. J. Appl. Res. Mem. Cogn. 6(4), 353-369 (2017)

25. Vijaykumar, S., Nowak, G., Himelboim, I., Jin, Y.: Virtual Zika transmission after the first U.S. case: who said what and how it spread on Twitter. Am. J. Infect. Control 46(5), 549557 (2018)

26. Jang, B., Jeong, S., Kim, C.: Distance-based customer detection in fake follower markets. Inf. Syst. 81, 104-116 (2019)

27. Borges-Tiago, M.T., Tiago, F., Cosme, C.: 'Exploring users' motivations to participate in viral communication on social media. J. Bus. Res. 101, 574-582 (2018)

28. Alzanin, S.M., Azmi, A.M.: Detecting rumors in social media: a survey. Proc. Comput. Sci. 142, 294-300 (2018)

29. Rayess, M.E., Chebl, C., Mhanna, J., Hage, R.-M.: Fake news judgement: the case of undergraduate students at Notre Dame University-Louaize, Lebanon. Ref. Serv. Rev. 46(1), 146-149 (2018)

30. Wessel, M., Thies, F., Benlian, A.: The emergence and effects of fake social information: evidence from crowdfunding. Decis. Supp. Syst. 90, 75-85 (2016) 
31. Ishida, Y., Kuraya, S.: Fake news and its credibility evaluation by dynamic relational networks: a bottom up approach. Proc. Comput. Sci. 126, 2228-2237 (2018)

32. Lee, S., Ha, T., Lee, D., Kim, J.H.: Understanding the majority opinion formation process in online environments: an exploratory approach to Facebook. Inf. Process. Manag. 54(6), 1115-1128 (2018)

33. Seargeant, P., Tagg, C.: Social media and the future of open debate: a user-oriented approach to Facebook's filter bubble conundrum. Discourse Context Media 27, 41-48 (2019)

34. De keersmaecker, J., Roets, A.: 'Fake news': incorrect, but hard to correct. The role of cognitive ability on the impact of false information on social impressions. Intelligence $\mathbf{6 5}$, 107-110 (2017)

35. Park, K., Rim, H.: Social media hoaxes, political ideology, and the role of issue confidence. Telematics Inform. 36, 1-11 (2019)

36. Weidner, K., Beuk, F., Bal, A.: Fake news and the willingness to share: a schemer schema and confirmatory bias perspective. J. Prod. Brand Manag. (2019). https://doi.org/10.1108/ JPBM-12-2018-2155

37. Mihaylov, T., Mihaylova, T., Nakov, P., Màrquez, L., Georgiev, G.D., Koychev, I.K.: The dark side of news community forums: opinion manipulation trolls. Internet Res. 28(5), 1292-1312 (2018)

38. Baccarella, C.V., Wagner, T.F., Kietzmann, J.H., McCarthy, I.P.: Social media? It's serious! Understanding the dark side of social media. Eur. Manag. J. 36(4), 431-438 (2018)

39. Zhang, Z., Gupta, B.B.: Social media security and trustworthiness: overview and new direction. Future Gener. Comput. Syst. 86, 914-925 (2018) 